Th-October, 1997

\title{
A Canonical Realization of the BMS Algebra
}

\author{
G.Longhi and M.Materassi \\ Department of Physics, University of Firenze
}

(July 12, 2018)

\begin{abstract}
A canonical realization of the BMS (Bondi-Metzner-Sachs) algebra is given on the phase space of the classical real Klein-Gordon field .

By assuming the finiteness of the generators of the Poincaré group, it is shown that a countable set of conserved quantities exists (supertranslations); this set transforms under a particular Lorentz representation, which is uniquely determined by the requirement of having an invariant fourdimensional subspace, which corresponds to the Poincaré translations. This Lorentz representation is infinite-dimensional, non unitary, reducible and indecomposable. Its representation space is studied in some detail. It determines the structure constants of the infinite-dimensional canonical algebra of the Poincaré generators together with the infinite set of the new conserved quantities.
\end{abstract}

It is shown that this algebra is isomorphic with that of the BMS group.

03.30.+p; 03.50.-z;04.20.Fy;11.10.-z

Typeset using REVTEX 


\section{INTRODUCTION}

The purpose of this paper is to give a canonical realization of the algebra of the BondiMetzner-Sachs (BMS) group [1] on the phase space of the real classical Klein-Gordon field.

The BMS group arises in general relativity as the asymptotic symmetry at null infinity of a space-time describing an isolated (radiating) gravitational source. Such space-time is expected to become flat at null infinity [2], and to exhibit the Poincaré group as the group of asymptotic symmetry.

While an asymptotic symmetry exists, its group is not the Poincaré group. It is a larger group containing the Poincaré group as a subgroup, and is a semidirect product of the homogeneous Lorentz group with an infinite dimensional abelian group. Among the infinitely many generators of this abelian group there are the generators of translations; the remaining are the so-called supertranslations.

The idea of asymptotic flatness was formalized by Penrose by a process of conformal compactification [5]; by means of this process a boundary (scri) was added to space-time, consisting of end points of null geodesics. In this way the notion of flatness acquired an intrinsic meaning.

The asymptotic symmetry at null infinity has been studied by several authors. Apart from the classic papers [1] we also quote some review articles [3]. Here we only mention some points discussed in the literature on this argument.

One problem with the BMS group is due to the presence of infinitely many copies of the homogeneous Lorentz group, obtained from one another by conjugation with a supertranslation. This happens even in the case of the Poincaré group, but the spin Casimir of the Poincaré group is not invariant under such conjugation 4 . This causes difficulties in the definition of a unique Poincaré group, and, by consequence, in the definition of the angular momentum of the system [6] (see however [7]).

The unitary and irreducible representations of the BMS group have been studied in [8] to give a possible interpretation of this group as a fundamental group of elementary particles, 
a line of research no longer pursued.

There is a situation analogous to that of null infinity in the case of spatial infinity, where a similar (bigger) asymptotic symmetry arises [9].

More recently the classical approach to null infinity was criticized [10], and it was shown that a more general asymptotic expansion for the metric should be used (polyhomogeneous expansion). Nevertheless, the group of asymptotic symmetry at null infinity is still the BMS group.

In view of the role played by the BMS group in general relativity it seems of interest to show that its algebra can be canonically realized in terms of conserved quantities of a real classical Klein-Gordon field. While this field has been chosen for its simplicity we expect that a similar analysis could be done for any other classical field. In particular, we will give explicitly the underlying infinite-dimensional representation of the Lorentz algebra, which determines the structure constants of the BMS algebra. Indeed we will show that, besides the total energy-momentum $P^{\mu}$ of the Klein-Gordon field, it is possible to define a countable set of other conserved quantities, with vanishing Poisson brackets with $P^{\mu}$ and among themselves. The requirement of the finiteness of $P^{\mu}$ implies a bound on the asymptotic behaviour of the Fourier coefficients of the field, when infinity is approached in the momentum space. This asymptotic behaviour is the same as required for the finiteness of this new set of conserved quantities.

More precisely, the Fourier coefficients of the field are defined on the mass hyperboloid, which is a three-dimensional Riemannian manifold with constant negative curvature. On this manifold the Laplace-Beltrami operator is naturally defined. It is the only non-vanishing Casimir operator of the Lorentz group on the space of functions on this manifold. It is by the study of the eigenfunctions of this operator that we discover the required representation of the Lorentz group, which, as we will see, is an infinite-dimensional, non-unitary and reducible representation.

The four functions $k^{\mu}$ on the mass hyperboloid are eigenfunctions of the Laplace-Beltrami operator. In addition there exists a countable set of eigenfunctions, belonging to the same 
eigenvalue, which are, like $k^{\mu}$, not square integrable, with respect to the Lorentz invariant measure. This set, together with $k^{\mu}$, transforms according to the representation, and, what is crucial, all these functions have the same asymptotic behaviour. The functions $k^{\mu}$ span an invariant four-dimensional subspace of this representation, but the orthogonal complement of this subspace is not invariant. So this representation is reducible but not decomposable.

The structure of this representation function space is the same as that of the translations and supertranslations of the BMS group. We show that the Poisson algebra of the conserved quantities built from these functions and the generators of the Lorentz group is the same as that of the BMS group. So we call these conserved quantities the generators of supertranslations.

The action of the Lorentz generators on the supertranslations defines a set of structure constants of the BMS algebra, which are the matrix elements of the infinite-dimensional representation of the Lorentz group. The action of the supertranslations on the field is non-local. Nevertheless it defines, as in the case of $P^{\mu}$, a canonical transformation on the field.

In Section II we recall some definitions about the Klein-Gordon field and define the Laplace-Beltrami operator on the mass hyperboloid. The eigenfunctions of this operator are studied in Section III and, in Section IV, the infinite representation of the Lorentz algebra is studied in some detail. In Section V the Poisson algebra of the generators of supertranslations is given and it is shown that it is isomorphic with the algebra of the BMS group.

The definitions and notations for the Klein-Gordon field are given in Appendix A. In Appendix B we discuss the eigenfunction problem for the Laplace-Beltrami operator on the mass hyperboloid. Moreover, we study in some detail the representation of the Lorentz group, which is the basis for the definition of the supertranslation generators. 


\section{THE KLEIN-GORDON FIELD AND THE LAPLACE-BELTRAMI OPERATOR}

The notations and definitions for the real Klein-Gordon field $\Phi(\vec{x}, t)$ are given in Appendix A. For each $t \in R$ the field $\Phi(\vec{x}, t)$ will be supposed to belong, together with its spatial and temporal derivatives, to $L^{2}\left(R^{3}\right)$, that is

$$
(\Phi(\cdot, t), \dot{\Phi}(\cdot, t)) \in H^{1}\left(R^{3}\right) \times L^{2}\left(R^{3}\right)
$$

where $\bar{\Phi}=\frac{\partial \Phi}{\partial t}$ and where $H^{1}\left(R^{3}\right)$ is the $W_{2}^{1}\left(R^{3}\right)$ Sobolev space [11]. This implies the existence of the total energy-momentum

$$
P^{\mu}=\int d^{3} x\left[\dot{\Phi}(\vec{x}, t) \partial^{\mu} \Phi(\vec{x}, t)-\frac{1}{2} \eta^{\mu 0}\left(\partial_{\nu} \Phi(\vec{x}, t) \partial^{\nu} \Phi(\vec{x}, t)-m^{2} \Phi^{2}(\vec{x}, t)\right)\right],
$$

or, in terms of the Fourier coefficients of the field $\Phi$

$$
P^{\mu}=\int \tilde{d k} k^{\mu} \bar{a}(\vec{k}) a(\vec{k})
$$

where the measure $\tilde{d k}$ is defined in Appendix A and $k^{0}=\omega(k)$. The energy-momentum $P^{\mu}$ is time-like and future oriented.

Apart the existence of a well defined total momentum $P^{\mu}$, we require the existence of the generators of the Lorentz group. This implies a more stringent condition on $\Phi$, or on $a(\vec{k})$. We require the additional condition: $\vec{\nabla} a(\vec{k}) \in L^{2}\left(R^{3}\right)$. From Eq.(2.3), with $\mu=0$, we have that $a(\vec{k}) \in L^{2}\left(R^{3}\right)$; with this additional condition we have that $a(\vec{k})$ is continuous and that has a vanishing limit when $|\vec{k}| \rightarrow \infty[12$.

From Eq.(2.3) we see that the asymptotic behaviour when $|\vec{k}| \rightarrow \infty$ of $a(\vec{k})$ must be

$$
|a(\vec{k})| \simeq|\vec{k}|^{-\frac{3}{2}-\epsilon}
$$

for any $\epsilon>0$.

This means that any integral like (2.3), with some regular function of $\vec{k}$ in place of $k^{\mu}$, and with the same degree of growth at infinite, will exist, as will be explicitly seen in Section VI. 
The scalar field $\Phi(x)$, where $x \equiv(\vec{x}, t)$, transforms under a Poincaré transformation $U(\Lambda, \alpha)$ as

$$
(U(\Lambda, \alpha) \Phi)(x)=\Phi\left(\Lambda^{-1}(x-\alpha)\right)
$$

This induces the transformation on $a(\vec{k})$

$$
(U(\Lambda, \alpha) a)(\vec{k})=a\left(\Lambda^{-1} \vec{k}\right) e^{i(k \cdot \alpha)}
$$

In this last equation the notation $\Lambda^{-1} \vec{k}$ has the meaning $\Lambda_{\cdot \nu}^{i} k^{\nu}, i=1,2,3$, where $k^{\circ}=$ $\sqrt{m^{2}+\vec{k}^{2}}$

The canonical action of the Poincaré generators on $a(\vec{k})$ is given by (see reference [11])

$$
\begin{gathered}
\left\{P^{\mu}, a(\vec{k})\right\}=i k^{\mu} a(\vec{k}), \\
\left\{M^{\prime \mu \nu}, a(\vec{k})\right\}=D_{\mu \nu} a(\vec{k}),\left\{M^{\prime \mu \nu}, \bar{a}(\vec{k})\right\}=D_{\mu \nu} \bar{a}(\vec{k}),
\end{gathered}
$$

where $M^{\prime \mu \nu}$ is defined in Eq. (A23) and

$$
D_{\mu \nu}=\left(\eta_{\mu}^{i} k_{\nu}-\eta_{\nu}^{i} k_{\mu}\right) \frac{\partial}{\partial k^{i}}
$$

The differential operators $D_{\mu \nu}$ satisfy the algebra

$$
\left[D_{\mu \nu}, D_{\rho \lambda}\right]=\eta_{\mu \rho} D_{\nu \lambda}+\eta_{\nu \lambda} D_{\mu \rho}-\eta_{\mu \lambda} D_{\nu \rho}-\eta_{\nu \rho} D_{\mu \lambda}
$$

We can now work with the field $a(\vec{k})$ and its complex conjugate $\bar{a}(\vec{k})$, which are defined on the mass hyperboloid. This is a Riemannian manifold which will be called $H_{3}^{1}$, following the notations of [13], where such manifolds are studied.

Indeed, if we define $H_{3}^{1}$ as the inclusion $\mathrm{f}$ of the submanifold $q^{2}-m^{2}=0$ with $q^{0}>0$, in the Minkowski space $M^{4}$ (with coordinates $q^{\mu}$ ), with $\mathrm{f}$ defined by

$$
f:\left\{k^{i}\right\} \rightarrow\left\{q^{\mu}\right\}, \quad \text { with } \quad q^{\circ}=\sqrt{m^{2}+\vec{k}^{2}}, \quad \text { and } \quad q^{i}=k^{i}, \quad(i=1,2,3),
$$

we get for the induced metric $\hat{\eta}$ 


$$
\hat{\eta}=f^{*} \eta
$$

the following expression

$$
\hat{\eta}=\hat{\eta}_{i j} d k^{i} d k^{j}
$$

with

$$
\hat{\eta}_{i j}=\frac{1}{\omega^{2}(k)} k^{i} k^{j}-\delta_{i j}, \quad(i, j=1,2,3) .
$$

The inverse of the matrix $\left\{\hat{\eta}_{i j}\right\}$ is

$$
\hat{\eta}^{i j}=-\left(\delta^{i j}+\frac{k^{i} k^{j}}{m^{2}}\right)
$$

its determinant is

$$
|\hat{\eta}|=\operatorname{det}\left\{\hat{\eta}_{i j}\right\}=-\frac{m^{2}}{\omega^{2}(k)},
$$

and its eigenvalues are $-1,-1,-1+\frac{\vec{k}^{2}}{\omega^{2}(k)}$.

So the metric $\hat{\eta}$ is proper Riemannian. It can be shown that the manifold $H_{3}^{1}$ has constant negative curvature and that it is a space-like surface, whose normal is

$$
n^{\mu} \equiv\left(\frac{\omega(k)}{m}, \frac{\vec{k}}{m}\right) \equiv \frac{k^{\mu}}{m}, \quad n^{2}=1
$$

This normal can be extended to a vector field on the Minkowski space $M^{4}$ with

$$
n_{q}=\frac{q^{\mu}}{\sqrt{q^{2}}} \frac{\partial}{\partial q^{\mu}}
$$

with $q^{0}>0$.

The only invariant second order differential operator on $H_{3}^{1}$ is the Laplace-Beltrami operator $\Delta$ (no invariant differential operator of first order exists [16]):

$$
\Delta=\frac{1}{\sqrt{|\hat{\eta}|}} \frac{\partial}{\partial k^{i}} \hat{\eta}^{i j} \sqrt{|\hat{\eta}|} \frac{\partial}{\partial k^{j}}
$$

where $|\hat{\eta}|=\operatorname{det}\left\{\hat{\eta}_{i j}\right\}$.

Explicitly, this operator is 


$$
\Delta=-\frac{\omega(k)}{m} \frac{\partial}{\partial k^{i}}\left(\delta^{i j}+\frac{k^{i} k^{j}}{m^{2}}\right) \frac{m}{\omega(k)} \frac{\partial}{\partial k^{j}},
$$

or

$$
\Delta=-\left[\vec{\nabla}^{2}+\frac{2}{m^{2}} \vec{k} \cdot \vec{\nabla}+\frac{1}{m^{2}}(\vec{k} \cdot \vec{\nabla})^{2}\right]
$$

This operator is invariant under a Lorentz transformation $\Lambda$

$$
k^{i} \rightarrow k^{\prime i}=\Lambda_{j}^{i} k^{j}+\Lambda_{0}^{i} \sqrt{m^{2}+\vec{k}^{2}}
$$

The operator $\Delta$ is formally selfadjoint with respect to the invariant measure $\tilde{d k}$. It is an elliptic operator and has the property

$$
\Delta k^{\mu}=-\frac{3}{m^{2}} k^{\mu}
$$

Let us define

$$
D=m^{2} \Delta+3
$$

so that

$$
D k^{\mu}=0
$$

When explicitly written (see the following Section) the equation

$$
D f(\vec{k})=0
$$

is an equation which can be separated in spherical coordinates. The radial equation has three regular singularities in the complex plane of $z=-\frac{\vec{k}^{2}}{m^{2}}$, which are in $-1,0, \infty$, so it is a hypergeometric equation. The four functions $k^{\mu}$ are a subset of the solutions of Eq.(2.26), with the characteristic exponents $l=0,1$ in the neighborhood of the point $0\left(k^{\circ}=\omega(k)\right.$ has the exponent $l=0$ and $\vec{k}$ has the exponent $l=1$ ). In the neighborhood of the point at infinite the characteristic exponents are +1 and -3 . So we may expect that an infinite set of solutions could have the same asymptotic behaviour like $k^{\mu}$, when $|\vec{k}| \rightarrow \infty$.

We will see in the next Section that this is indeed the case and that there is an infinite set of solutions of the Eq. 2.26) which gives a set of well defined integrals, when we put them in place of $k^{\mu}$ in Eq. (2.3). 


\section{THE EIGENFUNCTIONS OF THE LAPLACE-BELTRAMI OPERATOR}

The Laplace-Beltrami operator $\Delta$ of Eqs.(2.19) and (2.21) was studied in a series of papers by Raczka, Limic and Niederle [13], where is called $\Delta\left(H_{3}^{1}\right)$ (see Eq.(5.10) in the first reference of [13]). There it is shown that $\Delta$ has no discrete spectrum, but only a continuous one, and the basis of its generalized eigenfunctions is determined.

$\Delta$ can be identified with one of the Casimir operators of the Lorentz group, whose Lie algebra is defined in terms of the differential operators

$$
l_{\mu \nu}=i D_{\mu \nu}
$$

with $D_{\mu \nu}$ as in the Eq.(2.9).

To make contact with the notations used by Naimark in his book [14] we put

$$
L_{i}=l_{i}, \quad K_{i}=-l_{0 i}, \quad l_{i}=-\frac{1}{2} \epsilon_{i j k} l_{j k}, \quad(i, j, k=1,2,3)
$$

(in Naimark's notations:

$$
\left.H_{3}=L_{3} ; \quad H_{ \pm}=L_{ \pm}=L_{1} \pm i L_{2} ; \quad F_{3}=K_{3} ; \quad F_{ \pm}=K_{ \pm}=K_{1} \pm i K_{2} .\right)
$$

These operators satisfy the Lorentz algebra

$$
\begin{aligned}
{\left[L_{i},\right.} & \left.L_{j}\right]=i \epsilon_{i j k} L_{k}, \\
{\left[K_{i}, L_{j}\right] } & =i \epsilon_{i j k} K_{k}, \\
{\left[K_{i}, K_{j}\right] } & =-i \epsilon_{i j k} L_{k} .
\end{aligned}
$$

Two invariant operators can be defined using $\vec{L}$ and $\vec{K}$

$$
\Xi_{1}=\vec{L} \cdot \vec{K}, \quad \Xi_{2}=|\vec{K}|^{2}-|\vec{L}|^{2}
$$

and these are the two Casimir operators of Lorentz group.

The operators $\vec{L}$ and $\vec{K}$ can be written

$$
\vec{L}=-i \vec{k} \wedge \vec{\nabla}, \quad \vec{K}=i \omega(k) \vec{\nabla}
$$


So

$$
\left\{\begin{array}{l}
\Xi_{1}=-[\vec{k} \wedge \vec{\nabla} \omega(k)] \cdot \vec{\nabla}, \\
\Xi_{2}=m^{2} \nabla^{2}+2(\vec{k} \cdot \vec{\nabla})+(\vec{k} \cdot \vec{\nabla})^{2}
\end{array}\right.
$$

$\omega(k)$ has a gradient which is parallel to $\vec{k}$, so $\Xi_{1}=0$. We are left with one Casimir only

$$
\Xi_{2}=\Delta
$$

In terms of $l_{\mu \nu}$ we have

$$
\Delta=-\frac{1}{2} l_{\mu \nu} l^{\mu \nu}, \quad \epsilon_{\mu \nu \rho \lambda} l^{\mu \nu} l^{\rho \lambda}=0
$$

and of course we have

$$
\left[D, D_{\mu \nu}\right]=0
$$

where the operator $D$ is defined in Eq.(2.24).

If we denote $\lambda$ the eigenvalue of $m^{2} \Delta$, the relation with Naimark's notations [14] is given by

$$
\lambda=-\left(k_{\circ}^{2}+c^{2}-1\right), \quad k_{\circ} c=0,
$$

where $k_{\circ}$ and $c$ are the eigenvalues of two operators $\Delta$ and $\Delta^{\prime}$ defined in the quoted reference

$$
\begin{array}{r}
\left.\Delta\right|_{\text {eigenvalue }}=-2\left(k_{\circ}^{2}+c^{2}-1\right) \\
\left.\Delta^{\prime}\right|_{\text {eigenvalue }}=-4 i k_{\circ} c .
\end{array}
$$

If we choose

$$
k_{\circ}=0, \quad c=i \Lambda,
$$

we get

$$
\lambda=1+\Lambda^{2} \in[1,+\infty)
$$


corresponding to the representations of the Lorentz group of the principal series, with $k_{\circ}=0$, which are unitary, see [14].

The zero modes of the operator $D$ of Eq.(2.24) correspond to

$$
\lambda=-3
$$

This value of $\lambda$ corresponds to a non unitary representation of the Lorentz group, which is reducible but not completely reducible as will be shown later. In Appendix B we give the details of the determination of this representation. Let us show here the expression of the boost $K_{3}$ and its action on the representation $\lambda=-3$. From Eqs.(3.1), (3.2) and (2.9) we get

$$
\begin{gathered}
l_{i j}=-\epsilon_{i j k} L_{k}=i\left(x^{i} \partial_{j}-x^{j} \partial_{i}\right), \\
l_{0 j}=-K_{j}=-i \sqrt{1+\vec{x}^{2}} \partial_{j},
\end{gathered}
$$

where

$$
\vec{x}=\frac{\vec{k}}{m}
$$

From these expressions we get

$$
K_{3}=i \sqrt{1+r^{2}}\left(\cos \theta \frac{\partial}{\partial r}-\frac{\sin \theta}{r} \frac{\partial}{\partial \theta}\right)
$$

in the spherical coordinates $(r, \theta, \phi)$ of $\vec{x}$.

$D$ becomes

$$
D=m^{2} \Delta+3=\left.\Delta\right|_{x}+3
$$

where

$$
\left.\Delta\right|_{x}=-\left[\left(1+r^{2}\right) \frac{\partial^{2}}{\partial r^{2}}+\left(\frac{2}{r}+3 r\right) \frac{\partial}{\partial r}-\frac{J^{2}}{r^{2}}\right]
$$

and $J^{2}=\vec{L}^{2}$ as usually. 
The eigenfunctions of $\left.\Delta\right|_{x}$ correspond to $\lambda \in[1,+\infty)$, or $\Lambda \in[0,+\infty)$ as a limit from the upper half-plane of the complex plane of $\Lambda$. They are given in [13] (also see Eqs.(B2) and (B3) )

$$
\begin{aligned}
u_{\lambda, l, m}(r, \theta, \phi) & =N_{\lambda l} v_{1, \lambda, l, m}^{(\circ)}= \\
& =N_{\lambda l} r^{l}{ }_{2} F_{1}\left(\frac{l+1+i \Lambda}{2}, \frac{l+1-i \Lambda}{2} ; l+\frac{3}{2} ;-r^{2}\right) Y_{l, m}(\theta, \phi),
\end{aligned}
$$

where ${ }_{2} F_{1}$ is the hypergeometric function, with the three parameters of ${ }_{2} F_{1}\left(\alpha, \beta ; \gamma ;-r^{2}\right)$ satisfying the relation

$$
\alpha+\beta+\frac{1}{2}=\gamma,
$$

and where $l=0,1,2, \ldots$, and $|m| \leq l$.

The normalization factor $N_{\lambda l}$ is

$$
N_{\lambda l}=\frac{2 \pi}{m \sqrt{\Lambda}}\left|\frac{\Gamma\left(\frac{l+2-i \Lambda}{2}\right) \Gamma\left(\frac{l+1-i \Lambda}{2}\right)}{\Gamma(i \Lambda) \Gamma\left(l+\frac{3}{2}\right)}\right| .
$$

for $\Lambda \in[0, \infty)$.

With this normalization $u_{\lambda, l, m}$ is an orthonormal set with respect to the scalar product of $L_{2}(\tilde{d k})$

$$
\int \tilde{d k} \bar{u}_{\lambda l m}(r, \theta, \phi) u_{\lambda^{\prime}, l^{\prime}, m^{\prime}}(r, \theta, \phi)=\delta_{l l^{\prime}} \delta_{m m^{\prime}} \delta\left(\Lambda-\Lambda^{\prime}\right) .
$$

The action of the generators $K_{3}$ and $L_{i}$ on this basis is

$$
K_{3} u_{\lambda, l, m}=-i|l+1+i \Lambda| C_{l+1, m} u_{\lambda, l+1, m}+i|l+i \Lambda| C_{l, m} u_{\lambda, l-1, m},
$$

where the last term in the r.h.s. is zero when $l=0$ and $|m|$ is always bounded by $l$ and, in the last term, by $l-1$;

$$
C_{l, m}=\sqrt{\frac{(l+m)(l-m)}{(2 l-1)(2 l+1)}},
$$

and

$$
\begin{aligned}
L_{3} u_{\lambda, l, m} & =m u_{\lambda, l, m}, \\
L_{ \pm} u_{\lambda, l, m} & =\sqrt{l(l+1)-m(m \pm 1)} u_{\lambda, l, m \pm 1},
\end{aligned}
$$


with

$$
L_{ \pm}=L_{1} \pm i L_{2}
$$

The action of $K_{1}, K_{2}$ can be recovered from the commutators

$$
K_{ \pm}=K_{1} \pm i K_{2}= \pm\left[K_{3}, L_{ \pm}\right]
$$

Observe that, if we define

$$
K_{3} u_{\lambda, l, m}=A_{\lambda, l, m} u_{\lambda, l+1, m}+B_{\lambda, l, m} u_{\lambda, l-1, m}
$$

we have

$$
B_{\lambda, l, m}=\bar{A}_{\lambda, l-1, m}
$$

when $\Lambda$ is real.This is the hermiticity condition for the $K_{3}$ matrix on the $u_{\lambda, l, m}$ basis when $\lambda \in[1,+\infty)$.

This basis is complete in $L_{2}(\tilde{d k})$, that is

$$
\sum_{l \geq 0} \sum_{|m| \leq l} \int_{1}^{\infty} d \lambda u_{\lambda, l, m}(r, \theta, \phi) \bar{u}_{\lambda, l, m}\left(r^{\prime}, \theta^{\prime}, \phi^{\prime}\right)=\Omega(k) \delta^{3}\left(k-k^{\prime}\right) .
$$

The expressions given up to now, that is Eqs.(3.21), (3.23), (3.24), (3.25), (3.31) and Eq.(3.32) hold for $\Lambda \in[0,+\infty)$, or $\lambda \in[1,+\infty)$, which corresponds to the continuous spectrum of $\Delta$. Since we are interested in the case $\lambda=-3$, we need the relations analogous to Eq. 3.25) for a generic complex $\Lambda$, with $\operatorname{Im} \Lambda \geq 0$.

For a complex $\Lambda$, and in particular for an immaginary $\Lambda$, the expression of the normalization factor $N_{\lambda l}$ becomes meaningless. Moreover, as discussed in the Appendix B, for $\lambda=-3$ the functions $u_{\lambda, l, m}$ are no more normalizable.

Following the discussion of Appendix B the only set of solutions of Eq.2.26) which we will take into account in the following is the set $\left\{v_{1, \lambda, l, m}^{(\circ)}\right\}$, with $\lambda=-3$.

The action of $K_{3}$ on $v_{1, \lambda, l, m}^{(\circ)}$ is

$$
K_{3} v_{\lambda, l, m}^{(\circ)}=-i \frac{(l+1+i \Lambda)(l+1-i \Lambda)}{2 l+3} C_{l+1, m} v_{\lambda, l+1, m}^{\circ}+i(2 l+1) C_{l, m} v_{\lambda, l-1, m}^{\circ}
$$


as given in Eq. (B31).

The action of $K_{ \pm}$is given in Eq.(B32), where the dependence on $\lambda$ of the coefficients in the r.h.s. is determined by the action of $K_{3}$ of Eq.(3.33).

From this equation we see that, for complex values of $\Lambda$ in the upper half-plane, the first term in the r.h.s. of Eq.(3.33) vanishes for

$$
\Lambda=i(l+1)
$$

We may conclude that the only representation with an invariant subspace of dimension $\underline{4}$ is the representation with

$$
\Lambda=2 i, \quad \text { or } \quad \lambda=-3 .
$$

In the next Section we will study in more detail this representation.

\section{THE REPRESENTATION $\lambda=-3$}

In this Section we will study in some detail the representation $\lambda=-3$. For $\Lambda=2 i$ let us define

$$
w_{l, m}(r, \theta, \phi)=v_{-3, l, m}^{\circ}(r, \theta, \phi) .
$$

From Eq.(3.33) we have the action of $K_{3}$ on $w_{l, m}$ :

$$
K_{3} w_{l, m}=-i \frac{(l-1)(l+3)}{(2 l+3)} C_{l+1, m} w_{l+1, m}+i(2 l+1) C_{l, m} w_{l-1, m},
$$

where the coefficients $C_{l, m}$ are defined in Eq.(3.26), and where the values of $|m|$ in the right hand side are always limited by $l$ in the first term and by $l-1$ in the second term.

Explicitly $w_{l, m}(\vec{x})$ is given by

$$
w_{l, m}(r, \theta, \phi)=r_{2}^{l} F_{1}\left(\frac{l-1}{2}, \frac{l+3}{2} ; l+\frac{3}{2} ;-r^{2}\right) Y_{l, m}(\theta, \phi),
$$

where $r, \theta$ and $\phi$ are the spherical coordinates of $\vec{x}=\frac{\vec{k}}{m}$. 
Observe that for $l=0,1$

$$
\begin{array}{r}
w_{0,0}=\sqrt{1+r^{2}} Y_{0,0}=\frac{\omega(k)}{m} Y_{0,0} \\
w_{1, m}=r Y_{1, m}=\frac{|\vec{k}|}{m} Y_{1, m}
\end{array}
$$

which are the components of the 4 -vector $\frac{k^{\mu}}{m}$ in the spherical basis, with the Minkowski diagonal metric given by

$$
\tilde{\eta}=\frac{4 \pi m^{2}}{3}(3,-1,-1,-1)
$$

where $(l, m)=(0,0),(1,-1),(1,0),(1,-1)$ and

$$
\sum_{l=0,1} \sum_{|m| \leq l} \bar{w}_{l, m} \tilde{\eta}_{l m, l m} w_{l, m} \equiv \bar{w} \cdot \tilde{\eta} \cdot w=m^{2}
$$

It is important to observe that all the functions $w_{l, m}$ have the same asymptotic behaviour as $|\vec{k}| \rightarrow \infty$, and not only those for $l=0,1$. In other words we have an infinite sequence of zero modes of the operator $D$, all with the same asymptotic behaviour as $k^{\mu}$. This fact is explicitly shown by Eq.(B13).

The subspace spanned by the component $l=0,1$ of $w_{l, m}$ is invariant under the action of the generators of the Lorentz group. Indeed, from the Eq.(4.2), we have

$$
\begin{aligned}
K_{3} w_{0,0} & =\frac{i}{\sqrt{3}} w_{1,0}, \\
K_{3} w_{1, m} & =i \sqrt{3} \delta_{m, 0} w_{0,0} .
\end{aligned}
$$

Moreover, since

$$
K_{ \pm}= \pm\left[K_{3}, L_{ \pm}\right]
$$

and $L_{ \pm}$doesn't modify the value of $l$, even the action of $K_{ \pm}$leaves the subspace $l=0,1$ invariant.

It is the factor $l-1$ in the r.h.s. of Eq. (4.2) which is responsible of this fact. There are no other values of $\Lambda$, in the upper half of its complex plane, which could provide an invariant subspace of the same dimension. If we choose $\Lambda=i n$, with $n$ integer $>0$, we have 
an invariant subspace with a dimension which is determined by the maximum value of $l$, which is equal to $n-1$, as we can see from Eq.(3.33). In conclusion, the value $\lambda=-3$, or $\Lambda=2 i$, is determined by the requirement of the existence of an invariant subspace of dimension 4 .

The representation whose basis is $\left\{w_{l, m}\right\}$ is reducible, but not decomposable (not completely reducible). That it is reducible is clear, since the matrix of $K_{3}$, determined by Eq.(3.33), is block triangular. Indeed, if we define

$$
K_{3} w_{l, m}=w_{l^{\prime}, m}\left(K_{3}\right)_{l^{\prime}, l}
$$

we have

$$
\left(K_{3}\right)_{2,1}=0
$$

but

$$
\left(K_{3}\right)_{1,2} \neq 0
$$

Now, a representation like this is decomposable if there exists a similarity transformation defined by a matrix $\mathrm{S}$ of the form

$$
\left(\begin{array}{ll}
1 & Y \\
0 & 1
\end{array}\right),
$$

transforming all the generators of the Lorentz group $l_{\mu \nu}$ in a block diagonal form, see [15].

The matrix $Y$ has four rows and infinitely many columns and of course it must be non-zero. It should similarly transform in a block diagonal form also the matrix of $\vec{L}^{2}$, which is already in block diagonal form. This is clearly impossible. So the representation is indecomposable.

\section{THE SUPERTRANSLATIONS}

The presence of an entire series of zero modes of the operator $D$, with the same asymp-

totic behaviour as $|\vec{k}| \rightarrow \infty$, allows us to define the following set of integrals 


$$
P_{l, m}=\int \tilde{d k} w_{l, m}(\vec{k}) \bar{a}(\vec{k}) a(\vec{k}),
$$

where

$$
\bar{P}_{l, m}=(-1)^{m} P_{l,-m},
$$

and where the functions $w_{l, m}$ are given in Eq.(4.3).

As shown in Appendix B (see Eq.(B13)) all these integrals are well defined.

The canonical action of the generators of the Lorentz group $M^{\mu \nu}$ on the $P_{l, m}$ can be obtained from Eqs.(2.7) and (2.8); since from Eq.(2.9) we have

$$
\left\{\begin{array}{l}
D^{i j}=\left(k^{i} \frac{\partial}{\partial k^{j}}-k^{j} \frac{\partial}{\partial k^{i}}\right)=i \epsilon_{i j k} L_{k}, \\
D^{0 j}=\omega(k) \frac{\partial}{\partial k^{j}}=-i K_{j},
\end{array}\right.
$$

with the definitions (3.1) and (3.2), we get

$$
\begin{gathered}
\left\{M^{i, j}, P_{l, m}\right\}=-i \epsilon_{i j k} P_{l^{\prime}, m^{\prime}}\left(L_{k}\right)_{l^{\prime}, m^{\prime} ; l, m}, \\
\left\{M^{0, j}, P_{l, m}\right\}=i P_{l^{\prime}, m^{\prime}}\left(K_{j}\right)_{l^{\prime}, m^{\prime} ; l, m} .
\end{gathered}
$$

In these equations the matrices $\left\|L_{k}\right\|$ and $\left\|K_{j}\right\|$ are those given in Eqs.(B24), (B36) and (B37), and correspond to the representation $\lambda=-3$ of the Lorentz algebra.

Moreover we have

$$
\left\{P_{l, m}, P_{l^{\prime}, m^{\prime}}\right\}=0 .
$$

For $l=0,1$ the $P_{l, m}$ are the spherical components of $P^{\mu}$.

The Eqs.(5.4), (5.5) and (5.6) show that this algebra is infinite-dimensional, that the abelian subalgebra of the translations and supertranslations $\left\{P_{l, m}\right\}$ is a normal subalgebra, and that the factor algebra is isomorphic to the Lorentz algebra.

In order to show that this algebra is the same as that of the BMS group, we simply redefine the $P_{l, m}$ with

$$
\hat{P}_{l, m}=\nu_{l} P_{l, m},
$$


with $\nu_{l}$ satisfying the recurrence relation

$$
\nu_{l+1}=\frac{2 l+3}{l+3} \nu_{l}
$$

or

$$
\nu_{l}=\frac{(2 l+1) ! !}{(l+2) !} 2 \nu_{0}
$$

By defining

$$
R_{z}=i K_{3}, \quad R_{ \pm}=i K_{ \pm}, \quad L_{z}^{\prime}=i L_{z}
$$

we get exactly the algebra given by Sachs [4] (see in this reference the Eqs.(IV.19) and $(\mathrm{IV} .20))$.

As shown by Sachs [4], the 4-dimensional subgroup of translations $\left(P_{l, m}\right.$ with $\left.l=0,1\right)$ is unique. On the other hand the homogeneous Lorentz group is not similarly unique. This is due to the fact that copies of the Lorentz group can be obtained by a conjugation with an arbitrary supertranslation.

Indeed, let us define the following transformation of the Lorentz generators

$$
\begin{gathered}
M^{\prime i, j} \rightarrow M^{\prime i, j}+\alpha\left\{P_{l, m}, M^{\prime i, j}\right\}= \\
=M^{\prime i, j}+i \alpha \epsilon_{i j k} P_{l^{\prime}, m^{\prime}}\left(L_{k}\right)_{l^{\prime}, m^{\prime} ; l, m}, \\
M^{\prime 0, j} \rightarrow M^{\prime 0, j}+\alpha\left\{P_{l, m}, M^{\prime 0, j}\right\}= \\
=M^{\prime 0, j}-i \alpha P_{l^{\prime}, m^{\prime}}\left(L_{j}\right)_{l^{\prime}, m^{\prime} ; l, m},
\end{gathered}
$$

where $\alpha$ is an arbitrary real parameter, and Eqs.(5.4), (5.5) were used.

For a fixed supertranslation $P_{l, m}$ we may exponentiate the infinitesimal transformation (5.11), (5.12), getting the same r.h.s. terms:

$$
\begin{gathered}
e^{\alpha P_{l, m}} * M^{\prime i, j}=M^{i i, j}+i \alpha \epsilon_{i j k} P_{l^{\prime}, m^{\prime}}\left(L_{k}\right)_{l^{\prime}, m^{\prime} ; l, m}, \\
e^{\alpha P_{l, m}} * M^{\prime 0, j}=M^{\prime 0, j}-i \alpha P_{l^{\prime}, m^{\prime}}\left(L_{j}\right)_{l^{\prime}, m^{\prime} ; l, m} .
\end{gathered}
$$


In these equations the $*$ operation is defined by

$$
e^{A} * B=\sum_{n \geq 0} \frac{1}{n !} D_{A}^{n} B, \quad D_{A}=\{A, \cdot\} .
$$

This transformation corresponds to a conjugation of the Lorentz algebra with an arbitrary fixed supertranslation. It can be verified that the transformed algebra is again the Lorentz algebra.

As a consequence, the Casimir operator of the Poincaré group given by the square of the Pauli-Lubanski four-vector, which is invariant under the transformation (5.13) and (5.14) when $l=0,1$, will in general change 4 .

The canonical action of a fixed supertranslation on the field is determined by

$$
\left\{P_{l, m}, a(\vec{k})\right\}=i w_{l, m}(\vec{k}) a(\vec{k}), \quad\left\{P_{l, m}, \bar{a}(\vec{k})\right\}=-i w_{l, m}(\vec{k}) \bar{a}(\vec{k})
$$

which, for $l=0,1$, reduces to (2.7), written in spherical coordinates.

Since $P_{l, m}$ is not real $\left(\bar{P}_{l, m}=(-1)^{m} P_{l,-m}\right)$, it induces two different canonical transformations on the field $\Phi$, determined by its real and its imaginary part

$$
P_{l, m}=R_{l, m}+i I_{l, m}
$$

where

$$
\left\{R_{l, m}, a(\vec{k})\right\}=i \operatorname{Re}\left(w_{l, m}(\vec{k})\right) a(\vec{k}), \quad\left\{R_{l, m}, \bar{a}(\vec{k})\right\}=-i \operatorname{Re}\left(w_{l, m}(\vec{k})\right) \bar{a}(\vec{k}),
$$

and

$$
\left\{I_{l, m}, a(\vec{k})\right\}=i \operatorname{Im}\left(w_{l, m}(\vec{k})\right) a(\vec{k}), \quad\left\{I_{l, m}, \bar{a}(\vec{k})\right\}=-i \operatorname{Im}\left(w_{l, m}(\vec{k})\right) \bar{a}(\vec{k})
$$

Again these can be exponentiated for a fixed supertranslation, for instance

$$
e^{\alpha R_{l, m}} a(\vec{k})=e^{i \alpha \operatorname{Rew} w_{l, m}(\vec{k})} a(\vec{k}), \quad e^{\alpha R_{l, m}} \bar{a}(\vec{k})=e^{-i \alpha \operatorname{Rew} w_{l, m}(\vec{k})} \bar{a}(\vec{k}),
$$

and the analogous expressions for $I_{l, m}$.

The Eq.(5.20) defines a canonical transformation of the field, since the canonical Poisson brackets (A18) are invariant under such transformation. 
This transformation is non-local on the field $\Phi(\vec{x}, t)$. It is a particular case of a linear transformation

$$
(\Phi, \Pi) \rightarrow\left(\Phi^{\prime}, \Pi^{\prime}\right)
$$

realized as an integral transformation of convolution type. Indeed, $a(\vec{k})$ belongs to $L^{2}\left(R^{3}\right)$, and so is a tempered distribution, and the exponentials $\exp \left(i R e w_{l, m}\right)$ and $\exp \left(i \operatorname{Im} w_{l, m}\right)$ are tempered distributions too. The convolution product between their Fourier transforms is well defined. Indeed, the functions $w_{l, m}(\vec{k})$, being the solutions of a homogeneous elliptic equation, are infinitely differentiable [16]. They and all their derivatives are polynomially bounded, due to the bound (B13). So, the functions $w_{l, m}(\vec{k})$ and their exponentials $\exp \left(i R e w_{l, m}\right), \exp \left(i \operatorname{Im} w_{l, m}\right)$, are multipliers in $S^{\prime}\left(R^{3}\right)$, the space of tempered distributions on $R^{3}$ [17]. This implies that their Fourier transforms are convolutes [18, that is it exists their convolution with any tempered distribution. In conclusion we may write the relation among $(\Phi, \Pi)$ and $\left(\Phi^{\prime}, \Pi^{\prime}\right)$ as

$$
\begin{aligned}
& \Phi^{\prime}(\vec{x}, t)=\int d^{3} x^{\prime}\left[f\left(\vec{x}-\vec{x}^{\prime}\right) \Phi(\vec{x}, t)+g\left(\vec{x}-\vec{x}^{\prime}\right) \Pi\left(\vec{x}^{\prime}, t\right)\right] \\
& \Pi^{\prime}(\vec{x}, t)=\int d^{3} x^{\prime}\left[h\left(\vec{x}-\vec{x}^{\prime}\right) \Phi(\vec{x}, t)+k\left(\vec{x}-\vec{x}^{\prime}\right) \Pi\left(\vec{x}^{\prime}, t\right)\right]
\end{aligned}
$$

where the distributions $f, g$ and $h$ are defined by

$$
\begin{aligned}
& f(\vec{x})=\int \tilde{d k} \omega(k)\left(e^{i \alpha \operatorname{Rew}_{l, m}(\vec{k})+i \vec{k} \cdot \vec{x}}+c . c\right), \\
& g(\vec{x})=i \int \tilde{d k}\left(e^{i \alpha \operatorname{Rew}_{l, m}(\vec{k})+i \vec{k} \cdot \vec{x}}-c . c\right), \\
& h(\vec{x})=-i \int \tilde{d k} \omega^{2}(k)\left(e^{i \alpha \operatorname{Rew}_{l, m}(\vec{k})+i \vec{k} \cdot \vec{x}}-c . c\right), \\
& k(\vec{x})=f(\vec{x}) .
\end{aligned}
$$

where c.c means the complex conjugated. It is easily seen that the condition

$$
\int d^{3} y\left[f(\vec{x}-\vec{y}) k\left(\vec{x}^{\prime}-\vec{y}\right)-g(\vec{x}-\vec{y}) h\left(\vec{x}^{\prime}-\vec{y}\right)\right]=\delta^{3}\left(\vec{x}-\vec{x}^{\prime}\right)
$$

which must be satisfied if the transformation (5.22) has to be canonical, holds.

The transformation induced by $I_{l, m}$ is the same with the replacement $R e w_{l, m} \rightarrow I m w_{l, m}$. 
The transformation (5.22) changes the initial configuration of the field determined by the functions $a(\vec{k})$, to a new one, determined by the functions $a^{\prime}(\vec{k})=\exp \left(i \alpha \operatorname{Re}\left(w_{l, m}(\vec{k})\right)\right) a(\vec{k})$. As we have seen this transformation can change the spin content of the field, when $l \geq 2$.

As a conclusion we collect the Eqs.(5.4), (5.5) and (5.6), which give the Poisson algebra of the BMS group

$$
\begin{aligned}
& \left\{M^{\prime \mu \nu}, P_{l, m}\right\}=P_{l^{\prime}, m^{\prime}}\left(M^{\prime \mu \nu}\right)_{l^{\prime}, m^{\prime} ; l, m}, \\
& \left\{P_{l, m}, P_{l^{\prime}, m^{\prime}}\right\}=0,
\end{aligned}
$$

where, as given by Eqs(5.4) and (5.5)

$$
\begin{aligned}
& \left(M^{\prime i j}\right)_{l^{\prime}, m^{\prime} ; l, m}=-i \epsilon_{i j k}\left(L_{k}\right)_{l^{\prime}, m^{\prime} ; l, m}, \\
& \left(M^{\prime 0 j}\right)_{l^{\prime}, m^{\prime} ; l, m}=i\left(K_{j}\right)_{l^{\prime}, m^{\prime} ; l, m},
\end{aligned}
$$

with the matrix elements of $L_{k}$ and of $K_{j}$ given in Eqs.(B24), (B36) and (B37).

\section{CONCLUSIONS}

Following our initial purpose, we have found a Poisson algebra isomorphic to the BMS algebra, realized on the phase space of a real classical Klein-Gordon field.

The structure constants of this algebra are given by the matrix elements of an infinitedimensional representation of the Lorentz group, which is non unitary, reducible and indecomposable. We have given an explicit basis of functions for this representation, which are not normalizable in the sense of an $L_{2}$ space with Lorentz invariant measure, but for which we give an asymptotic bound.

The requirement of the existence of a four-dimensional invariant subspace selects this representation almost uniquely among all the possible representations of the Lorentz group.

Thus, we have shown that it is possible to realize the BMS algebra outside the general relativity context, in which it was originally discovered. This fact suggests a more general role of the BMS group. 
As it is well known the BMS group is the semidirect product of the Lorentz group with the finite set of translations and the infinite set of supertranslations. In the general relativity case, on the basis of physical arguments, the vanishing of the supertranslations is required [4] [3]. In the case of the same algebra realized in terms of the Klein-Gordon field this requirement should be a strong restriction on the possible field configurations, which could not have a clear justification.

As we have observed in the Introduction, we may expect that a similar analysis could be worked out for other classical massive fields. Something similar should also happen for a zero mass field, by performing a harmonic analysis on the $k_{\mu} k^{\mu}=0$ cone, where the Laplace-Beltrami operator changes significantly [22].

The existence of the supertranslations in the context of the Klein-Gordon field theory is a byproduct of a larger study on the search of a canonical set of collective and relative variables for a classical relativistic field, playng a role analogous to the center of mass and relative variables of a non relativistic system of particles. This study will be the argument of a forthcoming paper.

\section{ACKNOWLEDGMENTS}

The authors wish to thank L. Lusanna for many suggestions and enlightening discussions on the subject of this paper, for his useful criticism and for reading the manuscript. The authors are also indebted with K. Marathe for many suggestions and for reading the manuscript.

\section{APPENDIX A: NOTATIONS FOR THE CLASSICAL REAL KLEIN-GORDON FIELD}

We list in this appendix the various definitions concerning the real Klein-Gordon field. We will put $c=\hbar=1$, and the metric signature is $(+;-,-,-)$.

The Lagrangian and the lagrangian density are 


$$
L=\int d t \mathcal{L}, \quad \mathcal{L}=\frac{1}{2}\left(\partial_{\mu} \Phi \partial^{\mu} \Phi-m^{2} \Phi^{2}\right),
$$

where $\mu=0,1,2,3$ and

$$
\bar{\Phi}=\Phi .
$$

The conjugate momentum and the equation of motion are

$$
\Pi(x)=\dot{\Phi}(x) \equiv \partial_{\circ} \Phi(x), \quad\left(\square+m^{2}\right) \Phi(x)=0 .
$$

The Nöether currents associated to a Poincaré transformation are given by

$$
\begin{aligned}
j^{\mu \nu} & =\partial^{\mu} \Phi \partial^{\nu} \Phi-\eta^{\mu \nu} \mathcal{L}=j^{\nu \mu}, \\
j^{\mu 0} & =\dot{\Phi} \partial^{\mu} \Phi=\Pi \partial^{\mu} \Phi, \quad(\mu \neq 0), \\
j^{00} & =\frac{1}{2}\left[\dot{\Phi}^{2}+(\vec{\nabla} \Phi)^{2}+m^{2} \Phi^{2}\right],
\end{aligned}
$$

and the generators of the Poincaré group are

$$
P^{\mu}=\int d^{3} x j^{0 \mu}(x), \quad M^{\mu \nu}=\int d^{3} x\left(x^{\mu} j^{0 \nu}-x^{\nu} j^{0 \mu}\right) .
$$

The canonical Poisson brackets are (see reference [11])

$$
\left\{\Phi\left(\vec{x}, x^{0}\right), \Pi\left(\vec{x}^{\prime}, x^{0}\right)\right\}=\delta^{3}\left(\vec{x}-\vec{x}^{\prime}\right),
$$

with the other Poisson brackets vanishing.

The Poincaré algebra is

$$
\begin{gathered}
\left\{P^{\mu}, P^{\nu}\right\}=0, \\
\left\{M^{\mu \nu}, P^{\rho}\right\}=P^{\mu} \eta^{\nu \rho}-P^{\nu} \eta^{\mu \rho}, \\
\left\{M^{\mu \nu}, M^{\rho \lambda}\right\}=M^{\mu \lambda} \eta^{\nu \rho}+\eta^{\mu \lambda} M^{\nu \rho}- \\
-M^{\mu \rho} \eta^{\nu \lambda}-\eta^{\mu \rho} M^{\nu \lambda} .
\end{gathered}
$$


The Fourier expansion of the field is

$$
\Phi(\vec{x}, t)=\int \tilde{d k}\left[a(\vec{k}) e^{-i(k \cdot x)}+\bar{a}(\vec{k}) e^{i(k \cdot x)}\right]
$$

where c.c. means the complex conjugate, and $(\cdot)$ is the usual Lorentz invariant scalar product between 4-vectors, and (we use the notations of [19])

$$
\begin{aligned}
\tilde{d k} & =\frac{d^{3} k}{\Omega(k)}, \quad \Omega(k)=(2 \pi)^{3} 2 \omega(\vec{k}), \\
\vec{k} & \equiv\left\{k^{i}\right\}, \quad(i=1,2,3), \quad k_{i}=-k^{i}, \\
\omega(k) & =k_{\circ}=\sqrt{\vec{k}^{2}+m^{2}} .
\end{aligned}
$$

If we denote with $\hat{\Phi}(\vec{k}, t)$ the Fourier transform of the field, with respect to the measure $d^{3} k$, then

$$
\Phi(\vec{k}, t)=\int d^{3} k \hat{\Phi}(\vec{k}, t) e^{i \vec{k} \cdot \vec{x}}, \quad \text { with } \quad \overline{\hat{\Phi}}(\vec{k}, t)=\hat{\Phi}(-\vec{k}, t)
$$

and

$$
a(\vec{k})=\frac{1}{2} \Omega(k) e^{i \omega(k) t}\left[\hat{\Phi}(\vec{k}, t)+\frac{i}{\omega(k)} \dot{\hat{\Phi}}(\vec{k}, t)\right] .
$$

From this we have the bound:

$$
|a(\vec{k})|^{2} \leq(2 \pi)^{6}\left[\vec{k}^{2}|\hat{\Phi}(\vec{k}, t)|^{2}+|\dot{\hat{\Phi}}(\vec{k}, t)|^{2}\right]
$$

Since we have assumed that $\Phi(\vec{x}, \cdot), \dot{\Phi}(\vec{x}, \cdot)$ and $\vec{\nabla} \Phi(\vec{x}, \cdot)$ be functions in $L^{2}\left(R^{3}\right)$, from the Parseval identity we get that $a(\vec{k}) \in L^{2}\left(R^{3}\right)$.

This implies the existence of $P^{\mu}$, but not of $M^{\mu \nu}$. This last it will be assured if we assume $\vec{\nabla} a(\vec{k}) \in L^{2}\left(R^{3}\right)$. So $a(\vec{k})$ will go to zero as $|\vec{k}| \rightarrow \infty$, as in Eq.(2.4).

The conjugate momentum is

$$
\Pi(x)=-i \int \tilde{d k} \omega(k)\left[a(\vec{k}) e^{-i(k \cdot x)}-\bar{a}(\vec{k}) e^{i(k \cdot x)}\right] .
$$

The Poisson brackets for the Fourier coefficients are

$$
\left\{a(\vec{k}), \bar{a}\left(\vec{k}^{\prime}\right)\right\}=-i \Omega(k) \delta^{3}\left(\vec{k}-\vec{k}^{\prime}\right),
$$


with the other Poisson brackets vanishing.

The Fourier coefficients in terms of the field are given by

$$
a(\vec{k})=\int d^{3} x e^{i(k \cdot x)}[\omega(\vec{k}) \Phi(x)+i \Pi(x)] .
$$

In terms of the Fourier coefficients the Poincaré generators are the following:

$$
\begin{gathered}
P^{\mu}=\int \tilde{d k} k^{\mu} \bar{a}(\vec{k}) a(\vec{k}), \\
M^{i j}=-i \int \tilde{d} k \bar{a}(\vec{k})\left(k^{i} \frac{\partial}{\partial k^{j}}-k^{j} \frac{\partial}{\partial k^{i}}\right) a(\vec{k})=\bar{M}^{i j}, \\
M_{0 j}=t P_{j}-i \int \tilde{d k} \bar{a}(\vec{k}) \omega(\vec{k}) \frac{\partial}{\partial k^{j}} a(\vec{k})=-M^{j o}=\bar{M}^{0 j} .
\end{gathered}
$$

We define

$$
\begin{aligned}
M^{\prime 0 j} & =-i \int \tilde{d k} \bar{a}(\vec{k}) \omega(k) \frac{\partial}{\partial k^{j}} a(\vec{k}), \\
M^{\prime i j} & =M^{i j},
\end{aligned}
$$

where $M^{\prime i j}$ and $M^{\prime 0 j}$ has the same Poisson algebra as $M^{i j}$ and $M^{0 j}$, and will be used as the Lorentz generators.

\section{APPENDIX B: THE EIGENFUNCTIONS OF THE LAPLACE-BELTRAMI OPERATOR AND THE ACTION OF THE LORENTZ GENERATORS}

\section{The eigenfunctions of the Laplace-Beltrami operator}

The eigenfunctions of the continuous spectrum of the Laplace-Beltrami operator, corresponding to $\lambda \in[1, \infty)$, have been studied in [13]. However, we are interested even in the non-normalizable solutions of the equation

$$
(\Delta-\lambda) v_{\lambda, l, m}=0
$$

for different values of $\lambda$. 
A fundamental system of solutions, in the neighborhood of the origin, that is for $r \simeq 0$, of the Eq.(B1) in spherical coordinates, is

$$
\begin{aligned}
& v_{1, \lambda, l, m}^{(\circ)}(\vec{r})=u_{1, \lambda, l}^{(\circ)}(r) Y_{l, m}(\theta, \phi), \\
& v_{2, \lambda, l, m}^{(\circ)}(\vec{r})=u_{2, \lambda, l}^{(\circ)}(r) Y_{l, m}(\theta, \phi),
\end{aligned}
$$

where $r=\frac{|\vec{k}|}{m}, Y_{l, m}$ are the spherical harmonics as defined in [20], and

$$
\begin{gathered}
u_{1, \lambda, l}^{(\circ)}(r)=r_{2}^{l} F_{1}\left(\frac{l+1+i \Lambda}{2}, \frac{l+1-i \Lambda}{2} ; l+\frac{3}{2} ;-r^{2}\right), \\
u_{2, \lambda, l}^{(\circ)}(r)=r_{2}^{-l-1}{ }_{2} F_{1}\left(-\frac{l+i \Lambda}{2},-\frac{l-i \Lambda}{2} ; \frac{1}{2}-l ;-r^{2}\right), \\
\left(u_{2, \lambda, l}^{(\circ)}(r)=u_{1, \lambda,-l-1}^{(\circ)}(r)\right),
\end{gathered}
$$

The relation between $\lambda$ and $\Lambda$ is given by $\lambda=1+\Lambda^{2}$, with $\operatorname{Im} \Lambda \geq 0$, as in Section III. A fundamental system in the neighborhood of the point at infinite $r \simeq \infty$ is

$$
\begin{aligned}
& v_{1, \lambda, l, m}^{(\infty)}(\vec{r})=u_{1, \lambda, l}^{(\infty)}(r) Y_{l, m}(\theta, \phi), \\
& v_{2, \lambda, l, m}^{(\infty)}(\vec{r})=u_{2, \lambda, l}^{(\infty)}(r) Y_{l, m}(\theta, \phi),
\end{aligned}
$$

where

$$
\begin{aligned}
& u_{1, \lambda, l}^{(\infty)}(r)=r^{-1-i \Lambda}{ }_{2} F_{1}\left(\frac{l+1+i \Lambda}{2},-\frac{l-i \Lambda}{2} ; 1+i \Lambda ;-\frac{1}{r^{2}}\right), \\
& u_{2, \lambda, l}^{(\infty)}(r)=r^{-1+i \Lambda}{ }_{2} F_{1}\left(\frac{l+1-i \Lambda}{2},-\frac{l+i \Lambda}{2} ; 1-i \Lambda ;-\frac{1}{r^{2}}\right) .
\end{aligned}
$$

For $r \geq 0$ no other singular points are met. For the argument $z=-1$, the hypergeometric series is absolutely convergent, since the coefficients of ${ }_{2} F_{1}(\alpha, \beta ; \gamma ; z)$ satisfy the condition (see Eq. (3.22))

$$
\alpha+\beta-\gamma=-\frac{1}{2}
$$

If $i \Lambda$ is a positive integer, the solution $u_{1, \lambda, l}^{(\infty)}(r)$ requires a modification of the expression given in the Eq.(B6). However, since we are only interested in the solution $u_{1, \lambda, l}^{(\circ)}(r)$ for $\operatorname{Im} \Lambda \geq 0$, we do not give here the necessary modification. 
We are interested in the normalization properties of these solutions in the neighborhood of the points 0 and $\infty$, with respect to the invariant measure

$$
\tilde{d r}=\frac{d^{3} r}{\sqrt{1+r^{2}}}
$$

The solution $v_{1, \lambda, l, m}^{(\circ)}$ is regular and normalizable in the neighborhood of the origin. The solution $v_{2, \lambda, l, m}^{(\circ)}$ on the other hand is normalizable in the neighborhood of origin for $l=0,1$ only. We will discard this solution, even in the case $l=0,1$, since under the action of the Lorentz generators it will be transformed into a solution with a different value of $l$, thus becoming non-normalizable in the neighborhood of origin. See also the discussion at the end of subsection 2 .

The solution $u_{1, \lambda, l}^{(\circ)}$ can be analytically continued to $r \simeq \infty$, and, for a generic value of $\Lambda$ has an asymptotic expansion which is a linear combination of the two power of $r$ (see Eq. $2.10(2)$ of [21])

$$
r^{-1-i \Lambda}, \quad r^{-1+i \Lambda}
$$

and, for a real $\Lambda$, is normalizable in the sense of the continuous spectrum.

For real values of $\Lambda \in[0, \infty), v_{1, \lambda, l, m}^{(\circ)}$ is an eigenfunction of the operator $\Delta$, see [13], belonging to the continuous spectrum.

In the case $\lambda=-3$, that is $\Lambda=2 i$, we must use another asymptotic expansion. In general, for $\Lambda=n i$, with $\mathrm{n}$ integer, we must use the expansion given in Eq. 2.10(7) of [21]; for $\Lambda=2 i$ and for $r \rightarrow \infty$ we get

$$
u_{1,-3, l}^{(\circ)} \simeq r+O\left(r^{-1}\right)
$$

So, this solution is no more normalizable at $\infty$, but only at 0 .

The other solution at $r \simeq \infty$ has the behaviour

$$
u_{2,-3, l}^{(\circ)} \simeq r+O\left(r^{-1}\right), \quad \text { for } \quad l=0,1 ; \quad u_{2,-3, l}^{(\circ)} \simeq r+O\left(r^{-3}\right), \quad \text { for } \quad l \geq 2 .
$$

and is singular at the origin for $l>0$. So it is not normalizable at $r \simeq \infty$. 
As for the second fundamental system given in Eq. $(\mathrm{B} 5)$ and $(\mathrm{B} 6)$ they are linear combinations of the first set, since they can be obtained by analytic continuation using well known relations. The solution $u_{1, \lambda, l}^{(\infty)}$ has, for $r \simeq \infty$, the asymptotic behaviour

$$
r^{-1+\operatorname{Im} \Lambda-i \operatorname{Re} \Lambda}
$$

and, for $\operatorname{Im} \Lambda \geq 0$, is not normalizable.

The second solution $u_{2, \lambda, l, m}^{(\infty)}$ is instead normalizable for $r \simeq \infty$.

The solutions $w_{l, m}=v_{1,-3, l, m}^{(\circ)}$ satisfy the following inequality

$$
\left|w_{l, m}(\vec{k})\right| \leq \sqrt{\frac{2 l+1}{4 \pi}} \sqrt{1+r^{2}} M_{l},
$$

where

$$
\begin{aligned}
& M_{l}=\frac{\Gamma\left(l+\frac{3}{2}\right) \Gamma(2)}{\Gamma\left(\frac{l}{2}+2\right) \Gamma\left(\frac{l}{2}\right)}, \quad \text { if } \quad l \geq 2, \\
& M_{l}=1, \quad \text { if } \quad l=0,1 .
\end{aligned}
$$

Indeed, since

$$
\left|Y_{l, m}(\theta, \phi)\right| \leq \sqrt{\frac{2 l+1}{4 \pi}}
$$

and

$$
\begin{aligned}
u_{l}(r) & =r_{2}^{l} F_{1}\left(\frac{l-1}{2}, \frac{l+3}{2} ; l+\frac{3}{2} ;-r^{2}\right)= \\
& =r^{l}\left(1+r^{2}\right)^{\frac{1-l}{2}}{ }_{2} F_{1}\left(\frac{l-1}{2}, \frac{l}{2} ; l+\frac{3}{2} ; \frac{r^{2}}{1+r^{2}}\right),
\end{aligned}
$$

using Eq.2.9(3) of [21], and the bounds

$$
\begin{gathered}
0 \leq \frac{r^{2}}{1+r^{2}}<1 \\
\left|{ }_{2} F_{1}(\alpha, \beta ; \gamma ; x)\right| \leq F(\alpha, \beta ; \gamma ; 1)=\frac{\Gamma(\gamma-\alpha-\beta) \Gamma(\gamma)}{\Gamma(\gamma-\alpha) \Gamma(\gamma-\beta)},
\end{gathered}
$$

which hold if $\alpha, \beta, \gamma>0$ and $\gamma-\alpha-\beta>0$, we have from Eq.(B16) 


$$
\left|u_{l}(r)\right| \leq r^{l}\left(1+r^{2}\right)^{\frac{1-l}{2}} \frac{\Gamma(2) \Gamma\left(l+\frac{3}{2}\right)}{\Gamma\left(\frac{l}{2}+2\right) \Gamma\left(\frac{l+3}{2}\right)} .
$$

Now

$$
r^{l}\left(1+r^{2}\right)^{\frac{1-l}{2}} \leq \sqrt{1+r^{2}}
$$

and collecting the results we get the inequality (B13) for $l \geq 2$.

For $l=0,1$ the last hypergeometric function in Eq.(B16) is equal 1, and we get the inequality $(\overline{\mathrm{B} 13})$ for $l=0,1$.

The inequality $(\underline{\mathrm{B} 13})$ for $w_{l, m}$ implies the existence of the integrals $P_{l, m}$ defined in Eq.(5.1). Indeed

$$
\left|P_{l, m}\right| \leq \int \tilde{d k}\left|w_{l, m}(\vec{k})\right| \bar{a}(\vec{k}) a(\vec{k}) \leq \sqrt{\frac{2 l+1}{4 \pi}} M_{l} \frac{P_{\circ}}{m} .
$$

So, if $P_{\circ}$ exists, all the integrals $P_{l, m}$ will similarly exist.

\section{The action of the Lorentz generators}

Let us determine the action of the generators of the Lorentz group on the solutions $v^{(\circ)}$. The explicit expression of the generators is

$$
\begin{aligned}
L_{3} & =-i \frac{\partial}{\partial \phi} \\
L_{ \pm} & =L_{1} \pm i L_{2}=e^{ \pm i \phi}\left( \pm \frac{\partial}{\partial \theta}+i \cot \theta \frac{\partial}{\partial \phi}\right) \\
K_{3} & =i \sqrt{1+r^{2}}\left(\cos \theta \frac{\partial}{\partial r}-\frac{\sin \theta}{r} \frac{\partial}{\partial \theta}\right), \\
K_{ \pm} & =i \sqrt{1+r^{2}} e^{ \pm i \phi}\left(\sin \theta \frac{\partial}{\partial r}+\frac{\cos \theta}{r} \frac{\partial}{\partial \theta} \pm \frac{i}{r \sin \theta} \frac{\partial}{\partial \phi}\right) .
\end{aligned}
$$

They satisfy the algebra

$$
\begin{gathered}
{\left[L_{+}, L_{-}\right]=2 L_{3}, \quad\left[L_{3}, L_{ \pm}\right]= \pm L_{ \pm}} \\
{\left[K_{+}, K_{-}\right]=-2 L_{3}, \quad\left[K_{3}, K_{ \pm}\right]=\mp L_{ \pm}} \\
{\left[K_{3}, L_{3}\right]=0, \quad\left[K_{3}, L_{ \pm}\right]= \pm K_{ \pm}} \\
{\left[L_{3}, K_{ \pm}\right]= \pm K_{ \pm}, \quad\left[K_{ \pm}, L_{ \pm}\right]=0}
\end{gathered}
$$


The action of $L_{3}$ and $L_{ \pm}$is the usual one

$$
\begin{aligned}
L_{3} v_{. ., \lambda, l, m} & =m v_{. ., \lambda, l, m}, \\
L_{ \pm} v_{. ., \lambda, l, m} & =\sqrt{l(l+1)-m(m \pm 1)} v_{. ., \lambda, l, m \pm 1} .
\end{aligned}
$$

For the action of $K_{3}$ and $K_{ \pm}$we will use the following formulas

$$
\begin{gathered}
\frac{d}{d r} F\left(a_{l}, b_{l} ; c_{l} ;-r^{2}\right)=-\frac{r}{2 \sqrt{1+r^{2}}} \frac{\left(2 a_{l}\right)\left(2 b_{l}\right)}{c_{l}} F\left(a_{l+1}, b_{l+1} ; c_{l+1} ;-r^{2}\right), \\
\frac{d}{d r}\left[r^{l} F\left(a_{l}, b_{l} ; c_{l} ;-r^{2}\right)\right]= \\
=(2 l+1) \frac{r^{l-1}}{\sqrt{1+r^{2}}} F\left(a_{l-1}, b_{l-1} ; c_{l-1} ;-r^{2}\right)- \\
-(l+1) r^{l-1} F\left(a_{l}, b_{l} ; c_{l} ;-r^{2}\right),
\end{gathered}
$$

where $F\left(a_{l}, b_{l} ; c_{l} ;-r^{2}\right)$ is the hypergeometric function and where, see Eq.(3.24)

$$
a_{l}+b_{l}+\frac{1}{2}=c_{l}
$$

The Eq.(B25) can be obtained by using the Eqs.2.11(10) and 2.8(20) of [21], and the Eq.(B26) using the Eqs.2.8(27) and 2.11(10) of the same reference.

Moreover, we will use the properties of the spherical harmonics 20]

$$
\cos \theta Y_{l, m}(\theta, \phi)=C_{l+1, m} Y_{l+1, m}(\theta, \phi)+C_{l, m} Y_{l-1, m}(\theta, \phi),
$$

where

$$
C_{l, m}=\sqrt{\frac{(l-m)(l+m)}{(2 l-1)(2 l+1)}},
$$

and

$$
\begin{aligned}
& \sin \theta \frac{\partial}{\partial \theta} Y_{l, m}(\theta, \phi)=l \cos \theta Y_{l, m}(\theta, \phi)- \\
& -\sqrt{\frac{(2 l+1)\left(l^{2}-m^{2}\right)}{(2 l-1)}} Y_{l-1, m}(\theta, \phi) .
\end{aligned}
$$

With these relations we get 


$$
\begin{gathered}
K_{3} v_{1, \lambda, l, m}^{(\circ)}=-i \frac{(l+1+i \Lambda)(l+1-i \Lambda)}{(2 l+3)} C_{l+1, m} v_{1, \lambda, l+1, m}^{(\circ)}+ \\
+i(2 l+1) C_{l, m} v_{1, \lambda, l-1, m}^{(\circ)}
\end{gathered}
$$

and

$$
\begin{gathered}
K_{ \pm} v_{1, \lambda, l, m}^{(\circ)}= \pm i\left[\frac{(l+1+i \Lambda)(l+1-i \Lambda)}{(2 l+3)} \sqrt{\frac{(l+1 \pm m)(l+2 \pm m)}{(2 l+1)(2 l+3)}} v_{1, \lambda, l+1, m \pm 1}^{(\circ)}+\right. \\
\left.+(2 l+1) \sqrt{\frac{(l \mp m)(l \mp m-1)}{(2 l-1)(2 l+1)}} v_{1, \lambda, l-1, m \pm 1}^{(\circ)}\right]
\end{gathered}
$$

For the solution $v_{2, \lambda, l, m}$ we get similarly

$$
\begin{aligned}
K_{3} v_{2, \lambda, l, m}^{(\circ)}= & +i \frac{(l+i \Lambda)(l-i \Lambda)}{(2 l-1)} C_{l, m} v_{2, \lambda, l-1, m}^{(\circ)}- \\
& -i(2 l+1) C_{l+1, m} v_{2, \lambda, l+1, m}^{(\circ)}
\end{aligned}
$$

and

$$
\begin{aligned}
K_{ \pm} v_{2, \lambda, l, m}^{(\circ)}= \pm & i\left[\frac{(l+i \Lambda)(l-i \Lambda)}{(2 l-1)} \sqrt{\frac{(l \mp m)(l-1 \mp m)}{(2 l-1)(2 l+1)}} v_{2, \lambda, l-1, m \pm 1}^{(\circ)}+\right. \\
& \left.+(2 l+1) \sqrt{\frac{(l+1 \pm m)(l+2 \pm m)}{(2 l+1)(2 l+3)}} v_{2, \lambda, l+1, m \pm 1}^{(\circ)}\right] .
\end{aligned}
$$

If we put $\Lambda=2 i$ we get for

$$
\begin{gathered}
w_{l, m}=v_{1,-3, l, m}^{(\circ)}, \quad w_{l, m}^{\prime}=v_{2,-3, l, m}^{(\circ)}, \\
K_{3} w_{l, m}=-i \frac{(l-1)(l+3)}{(2 l+3)} C_{l+1, m} w_{l+1, m}+ \\
+i(2 l+1) C_{l, m} w_{l-1, m}, \\
K_{ \pm} w_{l, m}= \pm i\left[\frac{(l-1)(l+3)}{(2 l+3)} \sqrt{\frac{(l+1 \pm m)(l+2 \pm m)}{(2 l+1)(2 l+3)}} w_{l+1, m \pm 1}+\right. \\
\left.+(2 l+1) \sqrt{\frac{(l \mp m)(l \mp m-1)}{(2 l-1)(2 l+1)}} w_{l-1, m \pm 1}\right] . \\
K_{3} w_{l, m}^{\prime}=+i \frac{(l-2)(l+2)}{(2 l-1)} C_{l, m} w_{l-1, m}^{\prime}- \\
-i(2 l+1) C_{l+1, m} w_{l+1, m}^{\prime},
\end{gathered}
$$




$$
\begin{aligned}
K_{ \pm} w_{l, m}^{\prime}= & \pm i\left[\frac{(l-2)(l+2)}{(2 l-1)} \sqrt{\frac{(l \mp m)(l-1 \mp m)}{(2 l-1)(2 l+1)}} w_{l-1, m \pm 1}^{\prime}+\right. \\
& \left.+(2 l+1) \sqrt{\frac{(l+1 \pm m)(l+2 \pm m)}{(2 l+1)(2 l+3)}} w_{l+1, m \pm 1}^{\prime}\right]
\end{aligned}
$$

In the previous formulas, the terms on the right hand side with the function $w_{l, m}$ with $l$ negative or with $|m|>l$ are to be considered zero.

Referring to the Eq.(B36) and (B37), observe that the representation subspace corresponding to the values of $l=0,1$ is an invariant subspace. This is due to the factor $(l-1)$.

Similarly, looking at the Eq. (B38) and (B39), we see that the subspace corresponding to the values $l \geq 2$ is invariant.

Let us define the matrices corresponding to the representations $w_{l, m}$ and $w_{l, m}^{\prime}$

$$
M w_{l, m}=w_{l^{\prime}, m^{\prime}}(M)_{l^{\prime}, m^{\prime} ; l, m}, \quad M w_{l, m}^{\prime}=w_{l^{\prime}, m^{\prime}}^{\prime}\left(M^{\prime}\right)_{l^{\prime}, m^{\prime} ; l, m},
$$

where $M$ is any one of the Lorentz generators.

If we define the new bases

$$
\hat{w}_{l, m}=N_{l} w_{l, m}, \quad \text { and } \quad \hat{w}_{l, m}^{\prime}=N_{l}^{\prime} w_{l, m}^{\prime},
$$

with

$$
N_{l}=N_{l}^{\prime}=\frac{1}{\sqrt{2 l+1}}
$$

we get for the new matrices

$$
(\hat{M})_{l^{\prime}, m^{\prime} ; l, m}=\frac{N_{l}}{N_{l^{\prime}}}(M)_{l^{\prime}, m^{\prime} ; l, m}, \quad \text { and } \quad\left(\hat{M}^{\prime}\right)_{l^{\prime}, m^{\prime} ; l, m}=\frac{N_{l}}{N_{l^{\prime}}}\left(M^{\prime}\right)_{l^{\prime}, m^{\prime} ; l, m},
$$

the relation

$$
\left(\hat{M}^{\prime}\right)^{\dagger}=\hat{M}
$$

That is the representation $\hat{w}_{l, m}^{\prime}$ is the adjoint of the representation $\hat{w}_{l, m}$ [14] 


\section{REFERENCES}

[1] H. Bondi, M.G.J. Van der Burg and A.W.K. Metzner, Proc.R.Soc.Lond., A269, 21 (1962); R.K. Sachs, Proc.R.Soc. Lond., A270, 103 (1962); E.T.Newman and T.W.J.Unti, J.Math.Phys., 3, 891 (1062);

[2] For an exact definition of asymptotic flatness see for instance: R.M. Wald, "General Relativity", The Univ. of Chicago Press, 1984, Co. 11; J. Stewart, "Advanced general relativity", Cambridge Monographs on Mathematical Physics, Cambridge Univ. Press, 1991, Ch.3.

[3] R. Geroch, "Asymptotic Structure of Space-time", in Asymptotic Structure of Spacetime, E. Esposito and L. Witten (eds.), Plenum, 1977.

E.T. Newmann and K.P. Tod, "Asymptotically Flat Space-time", in General Relativity and Gravitation, Vol.2, Ed. H. Held, Plenum, New York, 1980.

R.K. Sachs, "Gravitational Radiation", and R.Penrose, "Conformal Treatment of Infinity", in Relativity groups and topology, Eds. C. De Witt and B. De Witt, Gordon and Breach, New York (1964).

J.Winicour, "Angular Momentum in General Relativity", in General Relativity and Gravitation, Vol. 2, Ed. A. Held (Plenum, New York, 1980).

B. Schmidt, "Asymptotic Flatness - a Critical Appraisal", in Advances in Gravitation and Cosmology, Wiley, 1993, p. 81.

H. Friedrich, "Asymptotic Structure of Space-time", in Recent Advances in General Relativity, A.I. Janis and J.R. Porter (eds.), Birkhauser, Basel, 1992, p. 146.

[4] R. Sachs, Phys.Rev. 128, 2851 (1962).

[5] R. Penrose, Phys.Rev.Lett., 10, 66 (1963); Proc.R.Soc.Lond., A284, 159 (1965); see also R. Penrose in reference [3]

[6] See J. Winicour in reference [3] 
[7] O.M. Moreschi, Class.Quantum Grav., 3, 503 (1986); J.Math.Phys., 31, 1208 (1990). J. Jezierski, "Bondi mass in classical field theory", gr-qc/9703083, 27 Mar. 97.

[8] V. Cantoni, J.Math.Phys., 7, 1361 (1966).

P.J. McCarthy, J.Math.Phys., 13, 1837 (1972); Phys.Rev.Lett., 29, 817 (1972); A330, 517 (1972); A333, 317 (1973); 335, 301 (1973).

[9] R. Arnowitt, S. Deser and C.W. Misner, Phys.Rev., 117, 1595 (1960); 118, 1100 (1960); 121, 1566 (1961); 122, 997 (1961).

P.G. Bergmann, Phys.Rev., 124, 274 (1961).

R. Geroch, J.Math.Phys., 13, 956 (1972).

A. Ashtekar and R.U. Hansen, J.Math.Phys., 19, 1542 (1978).

A. Ashtekar, "Asymptotic Structure of the Gravitational Field at Spatial Infinity", in General Relativity and Gravitation, Vol.2, Ed. A. Held, New York, Plenum, 1980.

[10] L. Andersson and P.T. Chruściel, Phys.Rev.Lett., 70, 2829 (1993).

P.T. Chruściel, M.A.H. MacCallum and D.B. Singleton, gr-qc/9305021, 25 May 93; see however P.G. Bergmann, Phys.Rev., 124, 274 (1961), where logarithmic terms were already considered.

[11] With this hypotheses the Klein-Gordon system is hamiltonian, see: P. Chernov and J.E. Mardsen, "Properties of Infinite Dimensional Hamiltonian Systems", Lecture Notes in Mathematics n. 425, eds. A. Dold and B. Eckmann, Springer-Verlag, Berlin, 1974.

See also: R. Schmid, "Infinite Dimensional Hamiltonian Systems", Monographs and Textbooks in Pkysical Science, Lecture Notes, ed. Bibliopolis, Na., 1987.

[12] See for instance R.D. Richtmyer, "Principles of Advanced Mathematical Physics", Springer-Verlag 1978, Vol.I, Ch.5.

[13] R. Raczka, N. Limic and J. Niederle, J.Math.Phys, 7, 1861 (1966);7, 2026 (1966); 8, 1079 (1967). See also: A.O. Barut and R. Raczka, "Theory of Group Representations 
and Applications", Polish Scient. Pub., Warszawa 1977, Ch.15.

[14] M.A. Naimark,"Les représentations linéaires du groupe de Lorentz", Dunod, Paris (1962).

[15] M. Naimark and A. Stern, "Théorie des représentations des groupes", Ed. de Moscou, MIR 1979.

[16] W. Rudin, "Functional Analysis", McGraw-Hill, 1991, Ch. 8.

[17] I.M. Gel'fand and G.E. Shilov, "Generalized Functions", Academic Press, New York and London, 1968, Vol.II, Ch. II.

[18] Same reference as in [17], Ch. III.

[19] C. Itzykson and J.B.Zuber, "Quantum Field Theory", Mc.Graw-Hill, 1980, Ch.3.

[20] A. Messiah, "Quantum Mechanics", North-Holland Pub. Comp., 1961, Vol.I, Appendix B.

[21] A. Erdélyi et al., "Higher Transcendental Functions", Vol. I, R.E.Krieger Pub.Comp., 1953, Reprint Edition 1985.

[22] See the second paper in reference 13 